\title{
Neuropsychiatric Symptoms in Primary Progressive Aphasia and Apraxia of Speech
}

\author{
Tarun D. Singh a Joseph R. Duffy ${ }^{a} \quad$ Edythe A. Strand ${ }^{a}$ \\ Mary M. Machulda ${ }^{b}$ Jennifer L. Whitwell ${ }^{c}$ Keith A. Josephs ${ }^{a}$ \\ Departments of a Neurology, ${ }^{b}$ Psychiatry and Psychology and ${ }^{\mathrm{C}}$ Neuroradiology, Mayo Clinic, \\ Rochester, Minn., USA
}

\section{Key Words}

Neuropsychiatric symptoms · Primary progressive aphasia - Progressive apraxia of speech

\begin{abstract}
Aim: To conduct a prospective analysis of the neuropsychiatric symptoms (NPS) across the three categories of primary progressive aphasia (PPA) and progressive apraxia of speech (PAOS), compare the prevalence and nature of the symptoms, and look at which symptoms could be helpful to better differentiate these PPA and PAOS categories. Methods: A total of 106 consecutive patients with a diagnosis of semantic variant $(n=13)$, logopenic variant $(n=$ $37)$, agrammatic variant $(n=15)$ or PAOS $(n=41)$ were included in this prospective study. The NPS were measured by the Neuropsychiatric Inventory Questionnaire. Results: There were 65 patients with PPA and 41 with PAOS diagnosis. The most distinguishing features between the two groups were anxiety, apathy, aberrant motor behavior and appetite, while among the subtypes of PPA they were disinhibition and appetite changes. PPA and PAOS patients initially exhibited depression, but with increased disease duration, PAOS patients showed apathy (55.5\%) while PPA patients showed disinhibition (28.6\%) and aberrant motor behavior (14.3\%). Conclusion: Mood symptoms like anxiety and appetite changes are more likely to be present at initial stages of PPA, whereas behavioral symptoms like aberrant motor behavior and apathy are likely to occur early in PAOS. The NPS seem to evolve with the progression of the disease in both PPA and PAOS.




\section{Introduction}

Primary progressive aphasia (PPA) is a group of neurodegenerative disorders, which present with language impairment as the most characteristic feature [1]. The diagnosis of PPA requires that an insidiously progressive language impairment should be the primary cognitive deficit for approximately 2 years after the symptom onset with no or minimal change in other cognitive functions including memory, visuospatial skills and executive abilities [2]. However, as the disease progresses, other cognitive domains may become impaired leading to PPA-plus, although the language impairment remains the most prominent anomaly [3]. A new classification was proposed in 2011, segregating the PPA patients into three categories based on their type of speech impairment [4]. The first category is known as the semantic variant (svPPA) and is characterized by anomia and impaired single word comprehension. The second is the logopenic variant (lvPPA), which is characterized by impaired single word retrieval and impaired repetition of phrases. The third is the agrammatic variant (agPPA), which includes patients with grammatical errors in written and verbal language. More recently, another category of patients with neurodegenerative speech disorders has been identified known as progressive apraxia of speech (PAOS). These patients present with a motor speech neurodegenerative disorder with speech impairment being the sole or dominant sign or symptom $[5,6]$.

Since PPA and PAOS are primarily neurodegenerative speech disorders, there has been a lot of research looking into the neuropathology and clinical features. However, little has been reported about the neuropsychiatric aspects. These aspects may be helpful as clinical markers for different stages and may help in categorizing patients between PPA and PAOS. There have been some studies looking at the neuropsychiatric symptoms (NPS) of PPA [7-10] but only one analyzed the symptoms according to PPA subtypes [10], and that study used an older PPA classification. Most of the previous studies also had relatively smaller cohorts. There have not been any studies looking into the neuropsychiatric aspects of PAOS patients.

In this study, we conducted a prospective analysis of the NPS across the three categories of PPA and PAOS to compare the prevalence and nature of the symptoms and look at the factors that may help us to better differentiate these categories.

\section{Methods}

Standard Protocol Approvals and Patient Consents

The study was approved by the Mayo Clinic Institutional Review Board, and all subjects signed consent forms for enrollment into the study.

\section{Patient Selection}

All patients who presented to the Mayo Clinic in Rochester, Minn., USA between July 2010 and March 2014 with a suspected speech and language disorder secondary to a neurodegenerative process were recruited prospectively. Subjects with concurrent illnesses that could account for language deficits or meeting criteria for other neurodegenerative syndrome were excluded. Only patients over the age of 18, with an informant to provide independent evaluation of functioning and who spoke English as their primary language were included. All patients underwent detailed speech and language examination, neurological evaluation, neuropsychological testing and neuroimaging analysis over a span of 48-72 h.

\section{Clinical Diagnostic Classification}

To be included in the study, patients must have had svPPA, lvPPA, agPPA or PAOS. Patients with PPA as a primary diagnosis and who were not able to be classified among any of the three categories (svPPA, lvPPA and agPPA) were not included in this study. Quantitative scores and video recordings of all the crucial aspects of the speech and language assessments were reviewed for all the study participants by 2 speech-language pathologists separately (J.R.D. and E.A.S.) for consensus agreement about the diagnosis. 
Singh et al.: Neuropsychiatric Symptoms in Primary Progressive Aphasia and Apraxia of Speech

Study participants who, by consensus agreement, demonstrated apraxia of speech (AOS) without, or with less prominent, aphasia, were classified as PAOS, based on the published criteria [5, 6]. Any participant who, by mutual agreement among the speech pathologists, demonstrated features of PPA was analyzed for the diagnostic features of each PPA subtype [4]. All participants had disrupted normal activities of daily living (talking on the phone, writing a letter) due to speech or language dysfunction. None complained of early deficits in other cognitive domains.

In accordance with the consensus guidelines for the classification of PPA, tasks for the evaluation of speech and language functions were qualitatively utilized for the assessment of the presence or absence of each diagnostic feature of the individual variants.

\section{Neuropsychiatric Examination}

All patients underwent detailed neurological examination by a behavioral and movement disorder specialist (K.A.J.) as well as standardized testing of cognitive, behavioral, functional and motor performance.

The NPS of all cases across different categories were measured by the Neuropsychiatric Inventory Questionnaire (NPI-Q) [11]. The NPI-Q measures and grades 12 behavioral domains according to the severity of the symptoms and the distress that is experienced by the caregiver due to those symptoms in the last 30 days. The distress index was not analyzed for this study. The severity of the symptoms was graded as $1-3$, with 1 being mild and 3 being severe. The 12 behavioral domains that are measured can be subcategorized as behavioral/compartmental (aberrant motor behavior, disinhibition, apathy/indifference), appetite/eating disorder, mood symptoms (anxiety, euphoria/elation, irritability/lability, depression) and disruptive/ psychotic symptoms (delusions, hallucinations, agitation/aggression, nighttime behavior). Adding the total score of the 12 behavioral domains yielded the total NPI score. In our study population, the NPI-Q testing was done by a behavioral neurologist (K.A.J.). The NPI-Q has been shown to be a reliable and valid scale for testing NPS $[11,12]$.

\section{Statistical Analysis}

Statistical analysis was performed using JMP 9.0.1 (SAS Institute Inc., Cary, N.C., USA). The analysis was done at the conventional two-tailed $\alpha$ level of 0.05 . Group differences for categorical variables were assessed with the $\chi^{2}$ test and Fisher's exact test for small numbers. Differences in the continuous variables were assessed using the Kruskal-Wallis one-way analysis of variance. Mann-Whitney U post hoc testing was performed if Kruskal-Wallis testing was significant. Multivariate regression analysis was used to compare the two groups of PPA and PAOS, and the odds ratio (OR) and 95\% confidence interval (CI) were computed. Demographics and neuropsychological profiles were summarized as percentages and means \pm SD.

\section{Results}

We included 106 patients [56 (52.8\%) males and 50 (47.2\%) females] with a mean age at onset of $63.9 \pm 8.1$ years who fulfilled the above criteria for the diagnosis of either PPA or PAOS during the study period. The mean age at testing was $66.8 \pm 8$ years, and their MiniMental State Examination (MMSE) score was $27.3 \pm 2.5$. After the final segregation, there were 65 patients with PPA and 41 with PAOS diagnosis. When the PPA patients were further categorized in accordance with the latest guidelines [4], it yielded 15 (23.1\%) with agPPA, 37 (56.9\%) with lvPPA and $13(20.0 \%)$ with svPPA diagnosis. The only significant difference between PPA and PAOS was observed on MMSE, with patients with PAOS presenting with a significantly higher mean MMSE. However, MMSE and illness duration were different among the various subtypes of PPA. The breakdown of the demographic variables, education, MMSE, age at onset and testing according to the patients' final diagnosis is presented in table 1 . The demographics according to the subtypes of PPA are presented in table 2.

There were no significant differences in the NPS between the PPA and PAOS patient groups when compared according to the severity of the NPI score. The frequency of distribution of NPS across PPA and PAOS according to the NPI breakdown is shown in table 3 and figure 1. However, significant differences became more apparent when the two groups were 
Dementia

Cognitive Disorders

Table 1. Demographics according to diagnosis

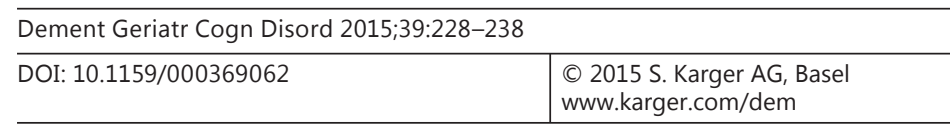

Singh et al.: Neuropsychiatric Symptoms in Primary Progressive Aphasia and Apraxia of Speech

\begin{tabular}{lccc}
\hline & PPA & PAOS & p value \\
\hline Patients, n & $65(61.3 \%)$ & $41(38.7 \%)$ & \\
Males, n & $33(50.7 \%)$ & $23(56.1 \%)$ & 0.5922 \\
Right-handedness ${ }^{\mathrm{a}}, \mathrm{n}$ & $57(87.7 \%)$ & $35(85.4 \%)$ & 0.6091 \\
Age at onset, years & $62.3 \pm 7.4$ & $65 \pm 8.9$ & 0.1092 \\
Illness duration, years & $3.2 \pm 2$ & $3.7 \pm 1.8$ & 0.1856 \\
Age at test, years & $65.5 \pm 7.4$ & $68.7 \pm 8.7$ & 0.0555 \\
MMSE score & $26.7 \pm 2.5$ & $28.3 \pm 2.1$ & 0.0005 \\
Education, years & $16 \pm 2.7$ & $15.3 \pm 2.7$ & 0.1545 \\
\hline
\end{tabular}

Continuous variables are presented as means \pm SD.

${ }^{a}$ One patient in the PAOS cohort was ambidextrous.

\begin{tabular}{lccrl}
\hline & agPPA & IvPPA & \multicolumn{1}{c}{ svPPA } & p value \\
\hline Patients, n & $15(14.2 \%)$ & $37(34.9 \%)$ & $13(12.2 \%)$ & \\
Males, n & $6(40 \%)$ & $21(56.8 \%)$ & $6(46.2 \%)$ & 0.6762 \\
Right-handedness, $\mathrm{n}$ & $13(86.6 \%)$ & $34(91.9 \%)$ & $10(76.9 \%)$ & 0.6801 \\
Age at onset, years & $63.6 \pm 8.6$ & $62.1 \pm 7.3$ & $61.7 \pm 6.5$ & 0.357 \\
Illness duration, years & $2.3 \pm 1.4$ & $3.2 \pm 1.5$ & $4.2 \pm 3.4$ & $0.014^{\mathrm{a}}$ \\
Age at test, years & $65.9 \pm 8.2$ & $65.3 \pm 7.6$ & $65.8 \pm 6.3$ & 0.2578 \\
MMSE score & $27.3 \pm 2.8$ & $26 \pm 2.3$ & $27.8 \pm 2.1$ & $0.025^{\mathrm{b}}$ \\
Education, years & $15.5 \pm 3.4$ & $15.9 \pm 2.5$ & $17 \pm 2.2$ & 0.228 \\
\hline \multicolumn{4}{c}{ Continuous variables are presented as means \pm SD. } & \\
\multicolumn{2}{c}{ a Significance achieved between svPPA and agPPA. } & \\
\multicolumn{2}{c}{ b Significance achieved between svPPA and lvPPA. } \\
\hline
\end{tabular}

Table 2. Demographics according to PPA subtypes

compared according to the presence or absence of the NPI-Q symptoms. The most distinguishing features between the two groups were anxiety $(p=0.0282)$, apathy $(p=0.0217)$, aberrant motor behavior $(p=0.0344)$ and appetite $(p=0.0068)$. The patients with PPA were 3.5 times more likely to have anxiety and 12.2 times more likely to have appetite abnormalities. However, apathy was 3.2 times and aberrant motor behavior 9.1 times more likely to be present in patients with PAOS. Delusions and euphoria were present only in the PPA patients, and hallucinations and nighttime behavior abnormalities were present in only $3(2.8 \%)$ and $6(5.7 \%)$ patients across both groups. Further details of the multivariate analysis comparing PPA and PAOS are shown in table 4.

When comparing the three subtypes of PPA (agPPA, lvPPA and svPPA), the most significant differentiating symptoms among them were disinhibition and appetite. Delusion and hallucination were present only in lvPPA, whereas aberrant motor behavior abnormalities were not present in this group. The details of NPS according to their severity among the three groups of PPA patients are presented in table 5.

\section{Discussion}

Our study with a large series of patients with PPA and PAOS - the largest collection of PAOS cases to date - highlights the behavioral differences between PPA and PAOS and among the three categories of PPAs. Mood symptoms like anxiety and appetite changes are more 
Dementia

Cognitive Disorders

Table 3. NPS in PPA and PAOS

\begin{tabular}{l|l}
\hline \multicolumn{2}{l|}{ Dement Geriatr Cogn Disord 2015;39:228-238 } \\
\hline DOI: 10.1159/000369062 & $\begin{array}{l}\text { @ 2015 S. Karger AG, Basel } \\
\text { www.karger.com/dem }\end{array}$ \\
\hline
\end{tabular}

Singh et al.: Neuropsychiatric Symptoms in Primary Progressive Aphasia and Apraxia of Speech

\begin{tabular}{|c|c|c|c|}
\hline & PPA & PAOS & $\mathrm{p}$ value \\
\hline Patients, $\mathrm{n}$ & $65(61.3 \%)$ & $41(38.7 \%)$ & \\
\hline NPI total & $2.8 \pm 2.9$ & $2.4 \pm 2.3$ & 0.5626 \\
\hline \multicolumn{4}{|l|}{ NPI breakdown } \\
\hline Delusion & & & 0.3737 \\
\hline 0 & $63(97 \%)$ & $41(100 \%)$ & \\
\hline 1 & $1(1.5 \%)$ & 0 & \\
\hline 2 & $1(1.5 \%)$ & 0 & \\
\hline Hallucination & & & 0.5815 \\
\hline 0 & $63(97 \%)$ & $40(97.6 \%)$ & \\
\hline 1 & $1(1.5 \%)$ & $1(2.4 \%)$ & \\
\hline 2 & $1(1.5 \%)$ & 0 & \\
\hline Agitation & & & 0.816 \\
\hline 0 & $53(81.6 \%)$ & $33(80.5 \%)$ & \\
\hline 1 & $8(12.3 \%)$ & $7(17.1 \%)$ & \\
\hline 2 & $3(4.6 \%)$ & $1(2.4 \%)$ & \\
\hline 3 & $1(1.5 \%)$ & 0 & \\
\hline Depression & & & 0.2018 \\
\hline 0 & 37 (57\%) & $28(68.3 \%)$ & \\
\hline 1 & $26(40 \%)$ & $10(24.4 \%)$ & \\
\hline 2 & $2(3 \%)$ & $3(7.3 \%)$ & \\
\hline Anxiety & & & 0.288 \\
\hline 0 & $41(63.1 \%)$ & 32 (78\%) & \\
\hline 1 & $17(26.2 \%)$ & $8(19.5 \%)$ & \\
\hline 2 & $6(9.2 \%)$ & $1(2.4 \%)$ & \\
\hline 3 & $1(1.5 \%)$ & 0 & \\
\hline Euphoria & & & 0.1593 \\
\hline 0 & $63(97 \%)$ & $41(100 \%)$ & \\
\hline 1 & $2(3.1 \%)$ & 0 & \\
\hline Apathy & & & 0.1179 \\
\hline 0 & $45(69.2 \%)$ & $21(51.2 \%)$ & \\
\hline 1 & $13(20 \%)$ & $15(36.6 \%)$ & \\
\hline 2 & $7(10.8 \%)$ & $4(9.8 \%)$ & \\
\hline 3 & 0 & $1(2.4 \%)$ & \\
\hline Disinhibition & & & 0.3967 \\
\hline 0 & $56(86.1 \%)$ & $39(95.1 \%)$ & \\
\hline 1 & $7(10.8 \%)$ & $2(4.9 \%)$ & \\
\hline 2 & $2(3.1 \%)$ & 0 & \\
\hline Irritability & & & 0.5229 \\
\hline 0 & 37 (57\%) & $22(53.7 \%)$ & \\
\hline 1 & $22(33.8 \%)$ & $16(39 \%)$ & \\
\hline 2 & $6(9.2 \%)$ & $2(4.9 \%)$ & \\
\hline 3 & 0 & $1(2.4 \%)$ & \\
\hline Aberrant motor behavior & & & 0.1544 \\
\hline 0 & $62(95.4 \%)$ & $36(87.8 \%)$ & \\
\hline 1 & $2(3.1 \%)$ & $4(9.8 \%)$ & \\
\hline 2 & $1(1.5 \%)$ & 0 & \\
\hline 3 & 0 & $1(2.4 \%)$ & \\
\hline Nighttime behavior & & & 0.6108 \\
\hline 0 & $61(93.9 \%)$ & $39(95.1 \%)$ & \\
\hline 1 & $3(4.6 \%)$ & $2(4.9 \%)$ & \\
\hline 2 & $1(1.5 \%)$ & 0 & \\
\hline Appetite & & & 0.0998 \\
\hline 0 & $53(81.5 \%)$ & $39(95.2 \%)$ & \\
\hline 1 & $9(1.4 \%)$ & $1(2.4 \%)$ & \\
\hline 2 & $3(4.6 \%)$ & $1(2.4 \%)$ & \\
\hline
\end{tabular}


Singh et al.: Neuropsychiatric Symptoms in Primary Progressive Aphasia and Apraxia of Speech

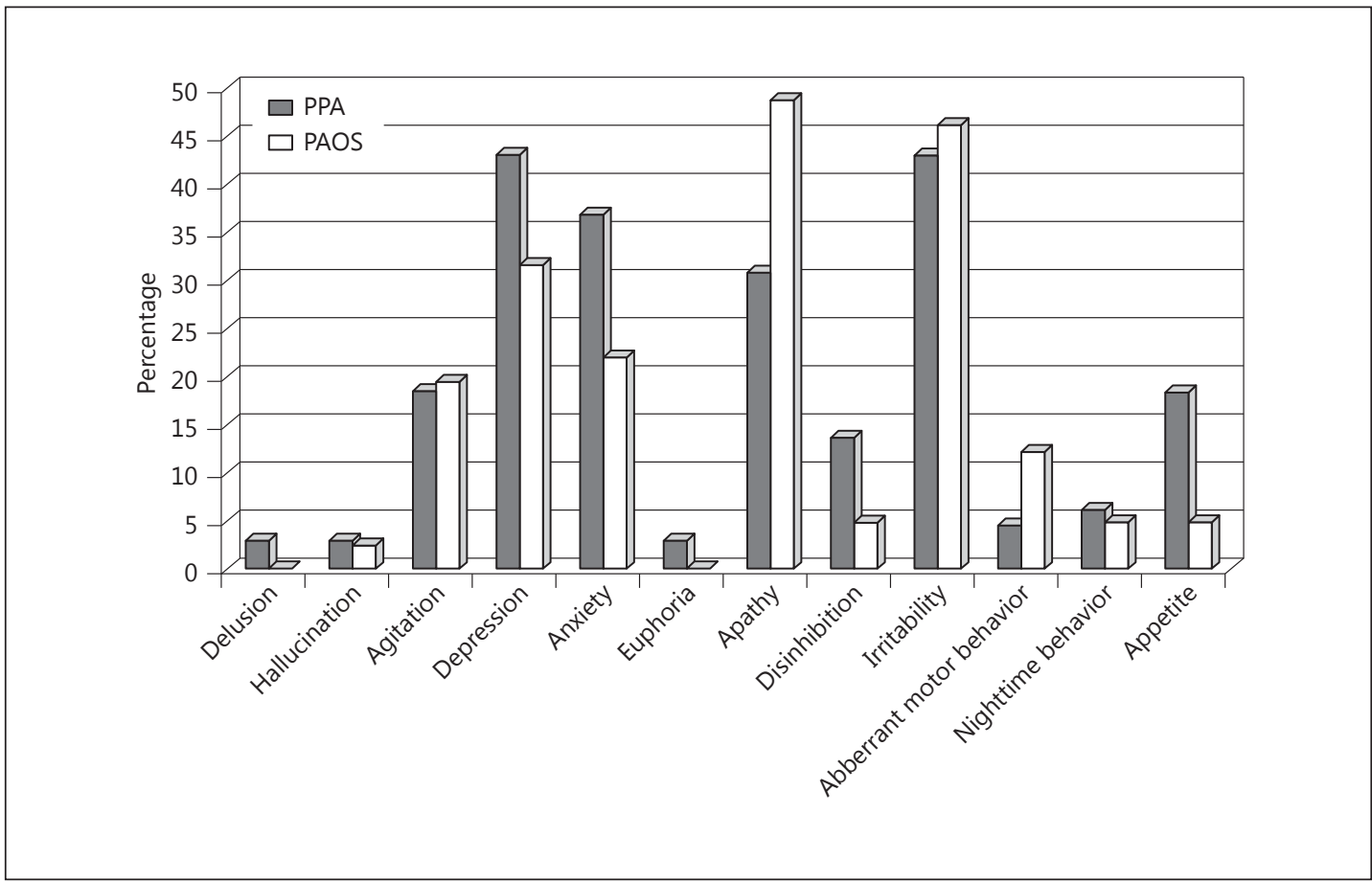

Fig. 1. Distribution of NPS across PPA and PAOS according to the NPI breakdown.

Table 4. Multivariate logistic regression analysis for NPI symptoms present or absent in PPA and PAOS

\begin{tabular}{lll}
\hline & OR $(95 \% \mathrm{CI})$ & p value \\
\hline Delusion & $\mathrm{N} / \mathrm{A}^{\mathrm{a}}$ & \\
Hallucination & $1.01(0.06-24.3)$ & 0.9963 \\
Agitation & $1.01(0.28-4.07)$ & 0.9484 \\
Depression & $1.33(0.49-3.63)$ & 0.57 \\
Anxiety & $3.55(1.14-12.65)$ & 0.0282 \\
Euphoria & $\mathrm{N} / \mathrm{A}^{\mathrm{a}}$ & \\
Apathy & $0.31(0.11-0.84)$ & 0.0217 \\
Disinhibition & $1.92(0.28-18.61)$ & 0.5176 \\
Irritability & $0.99(0.37-2.69)$ & 0.9869 \\
Aberrant motor behavior & $0.11(0.01-0.86)$ & 0.0344 \\
Nighttime behavior & $0.37(0.02-5.52)$ & 0.4717 \\
Appetite & $12.21(1.86-169.45)$ & 0.0068 \\
\hline
\end{tabular}

${ }^{\text {a }}$ Not applicable as symptoms were present only in one category.

likely to be present in PPA, whereas PAOS patients are more likely to have behavioral symptoms like aberrant motor behavior and apathy. Among the three categories of PPA, svPPA patients are more likely to show disinhibition, while appetite changes are more likely to be present in svPPA and agPPA patients.

AOS is a disorder of speech motor planning or programming that affects the production of speech [13]. More recently, AOS has been demonstrated in the context of neurodegenerative disorders $[6,13,14]$. In fact, a syndrome with motor speech impairment as the presenting sign in the absence of any aphasia is referred to as primary progressive apraxia of speech [6], 
Dementia

and Geriatric

Table 5. NPS among different PPA subtypes

\begin{tabular}{l|l}
\hline \multicolumn{2}{|l}{ Dement Geriatr Cogn Disord 2015;39:228-238 } \\
\hline DOI: $10.1159 / 000369062$ & $\begin{array}{l}\text { C 2015 S. Karger AG, Basel } \\
\text { www.karger.com/dem }\end{array}$ \\
\hline
\end{tabular}

Singh et al.: Neuropsychiatric Symptoms in Primary Progressive Aphasia and Apraxia of Speech

\begin{tabular}{|c|c|c|c|c|}
\hline & agPPA & IvPPA & svPPA & $\mathrm{p}$ value \\
\hline Patients, n & 15 & 37 & 13 & \\
\hline NPI total & $2.5 \pm 2.6$ & $2.6 \pm 2.9$ & $3.4 \pm 3.5$ & 0.6936 \\
\hline \multicolumn{5}{|l|}{ NPI breakdown } \\
\hline \multicolumn{4}{|l|}{ Delusion } & $\mathrm{N} / \mathrm{A}$ \\
\hline 0 & $15(100 \%)$ & 35 (94.6\%) & $13(100 \%)$ & \\
\hline 1 & 0 & $1(2.7 \%)$ & 0 & \\
\hline 2 & 0 & $1(2.7 \%)$ & 0 & \\
\hline \multicolumn{4}{|l|}{ Hallucination } & $\mathrm{N} / \mathrm{A}$ \\
\hline 0 & 15 (100\%) & 35 (94.6\%) & $13(100 \%)$ & \\
\hline 1 & 0 & $1(2.7 \%)$ & 0 & \\
\hline 2 & 0 & $1(2.7 \%)$ & 0 & \\
\hline \multicolumn{4}{|l|}{ Agitation } & 0.2607 \\
\hline 0 & $13(86.6 \%)$ & $30(81.1 \%)$ & $10(76.9 \%)$ & \\
\hline 1 & $1(6.7 \%)$ & $6(16.2 \%)$ & $1(7.7 \%)$ & \\
\hline 2 & $1(6.7 \%)$ & 0 & $2(15.4 \%)$ & \\
\hline 3 & 0 & $1(2.7 \%)$ & 0 & \\
\hline \multicolumn{4}{|l|}{ Depression } & 0.72 \\
\hline 0 & $10(66.7 \%)$ & $20(54.1 \%)$ & $7(53.8 \%)$ & \\
\hline 1 & $5(33.3 \%)$ & $16(43.2 \%)$ & $5(38.5 \%)$ & \\
\hline 2 & 0 & $1(2.7 \%)$ & $1(7.7 \%)$ & \\
\hline \multicolumn{4}{|l|}{ Anxiety } & 0.7167 \\
\hline 0 & $11(73.3 \%)$ & $23(62.2 \%)$ & $7(53.8 \%)$ & \\
\hline 1 & $3(20 \%)$ & $10(27 \%)$ & $4(30.8 \%)$ & \\
\hline 2 & $1(6.7 \%)$ & $4(10.8 \%)$ & $1(7.7 \%)$ & \\
\hline 3 & 0 & 0 & $1(7.7 \%)$ & \\
\hline \multicolumn{4}{|l|}{ Euphoria } & 0.413 \\
\hline 0 & $15(100 \%)$ & $36(97.3 \%)$ & $12(92.3 \%)$ & \\
\hline 1 & 0 & $1(2.7 \%)$ & $1(7.7 \%)$ & \\
\hline \multicolumn{4}{|l|}{ Apathy } & 0.7525 \\
\hline 0 & $10(66.7 \%)$ & $25(67.6 \%)$ & $10(76.9 \%)$ & \\
\hline 1 & $4(2.6 \%)$ & $8(21.6 \%)$ & $1(7.7 \%)$ & \\
\hline 2 & $1(6.7 \%)$ & $4(10.8 \%)$ & $2(15.4 \%)$ & \\
\hline \multicolumn{4}{|l|}{ Disinhibition } & $0.0245^{\mathrm{a}}$ \\
\hline 0 & $14(93.3 \%)$ & $34(91.9 \%)$ & $8(61.5 \%)$ & \\
\hline 1 & $1(6.7 \%)$ & $3(8.1 \%)$ & $3(23.1 \%)$ & \\
\hline 2 & 0 & 0 & $2(15.4 \%)$ & \\
\hline \multicolumn{4}{|l|}{ Irritability } & 0.4815 \\
\hline 0 & $8(53.3 \%)$ & $19(51.4 \%)$ & $10(76.9 \%)$ & \\
\hline 1 & $5(33.3 \%)$ & $15(40.5 \%)$ & $2(15.4 \%)$ & \\
\hline 2 & $2(13.4 \%)$ & $3(8.1 \%)$ & $1(7.7 \%)$ & \\
\hline \multicolumn{4}{|c|}{ Aberrant motor behavior } & 0.0542 \\
\hline 0 & $14(93.3 \%)$ & $37(100 \%)$ & $11(84.6 \%)$ & \\
\hline 1 & $1(6.7 \%)$ & 0 & $1(7.7 \%)$ & \\
\hline 2 & 0 & 0 & $1(7.7 \%)$ & \\
\hline \multicolumn{4}{|c|}{ Nighttime behavior } & 0.14 \\
\hline 0 & $13(86.7 \%)$ & $36(97.3 \%)$ & $12(92.3 \%)$ & \\
\hline 1 & $2(13.3 \%)$ & $1(2.7 \%)$ & $1(7.7 \%)$ & \\
\hline \multirow{2}{*}{\multicolumn{4}{|c|}{ Appetite }} & $0.0164^{\mathrm{a}}$ \\
\hline & & & & $0.0092^{\mathrm{c}}$ \\
\hline 0 & $10(66.7 \%)$ & $35(94.6 \%)$ & $8(61.5 \%)$ & \\
\hline 1 & $4(2.6 \%)$ & $2(5.4 \%)$ & $3(23.1 \%)$ & \\
\hline 2 & $1(6.7 \%)$ & 0 & $2(15.4 \%)$ & \\
\hline $\begin{array}{l}\text { N/A = Not a } \\
\text { a Significanc } \\
\text { b Significanc } \\
{ }^{c} \text { Significanc }\end{array}$ & $\begin{array}{l}\text { plicable. } \\
\text { achieved bet } \\
\text { achieved bet } \\
\text { achieved bet }\end{array}$ & $\begin{array}{l}\text { een lvPPA anc } \\
\text { een agPPA an } \\
\text { een lvPPA anc }\end{array}$ & $\begin{array}{l}\text { svPPA. } \\
\text { IvPPA. } \\
\text { svPPA. }\end{array}$ & \\
\hline
\end{tabular}


Singh et al.: Neuropsychiatric Symptoms in Primary Progressive Aphasia and Apraxia of Speech

which is subsumed under PAOS [5]. In our study, caregivers of PAOS most commonly endorsed apathy (48.8\%), irritability (46.3\%), depression $(31.7 \%)$, anxiety $(22 \%)$ and agitation (19.5\%). The less common symptoms were aberrant motor behavior (12.2\%), nighttime behavior abnormalities (4.9\%), appetite changes (4.9\%) and disinhibition (4.9\%). Delusion and euphoria were not present in any of our patients. PAOS patients are therefore more likely to demonstrate behavioral abnormalities like apathy and aberrant motor behavior abnormalities when compared to PPA patients. These findings are not unexpected as PAOS has often been linked to progressive supranuclear palsy (PSP) pathology [15], and profound apathy is often a common and supportive feature of PSP $[16,17]$. PAOS patients also demonstrate midbrain atrophy, which is a feature of pathologically confirmed PSP $[5,18]$. This association has been further quantified in a recent study showing the evolution of primary progressive apraxia of speech, which demonstrated that such patients may progress to a PSP-like syndrome referred to as PSP-AOS [19].

There have been some studies looking at the NPS of PPA patients [7-10]. However, none of them compared the various subtypes of PPA according to the latest classification. In our cohort of PPA patients, the most common presentation was lvPPA (56.9\%). The earliest age at onset of PPA was $61.7 \pm 6.5$ years in svPPA patients, whereas agPPA patients presented the earliest to the hospital ( $2.3 \pm 1.4$ years). Differentiating symptoms among the three subtypes of PPA were disinhibition ( $p=0.0245)$ and appetite changes $(\mathrm{p}=0.006)$. Disinhibition was more likely to be present in svPPA compared to the other two subtypes. Interestingly, although disinhibition is the most common in sVPPA compared to the other PPA variants and PAOS, it has been shown to be less common in semantic dementia compared to the behavioral variant of frontotemporal dementia [20]. The appetite changes were highly significant among the three groups, with svPPA and agPPA patients more likely to present with these changes. Depression was present in $43 \%$ of PPA patients, with the lvPPA diagnosis encompassing the majority of patients (60.7\%). Depression has also been observed in PPA patients [1, 21, 22]. Interestingly, depression was present in 4 of the 6 PPA patients that were first reported by Mesulam [1] in 1982.

In our study, delusion and hallucinations were present in only lvPPA among the PPA patients. This is a novel finding and could be due to the fact that lvPPA has been linked to the Alzheimer's disease pathology which can co-occur with Lewy body disease [23]. Lewy body disease is strongly associated with hallucinations. In fact, in a recent clinicopathological series, mixed Alzheimer's and Lewy body disease was reported in 8\% of lvPPA cases [24].

Some researchers have suggested that PAOS should not be considered different from patients with nonfluent/agPPA, although we have clearly demarcated that PAOS is anatomically different from agPPA with involvement of the bilateral superior and mid-premotor cortex [5], in contrast to agPPA, which affects the left posterior frontoinsular cortex [2]. The findings of this study further strengthen the argument of segregating them as the patients with PPA were more likely to have mood symptoms like anxiety and appetite changes, while PAOS patients are more likely to have behavioral symptoms like aberrant motor behavior and apathy.

The type of behavioral symptoms changed over time with longer disease durations. Patients with both PPA and PAOS showed symptoms initially inclined towards mood disorders like depression. This may be related to an emotional response towards the disease affecting their daily communication capabilities. However, with the increase in disease duration, PAOS patients more likely showed apathy (55.5\%), while PPA patients more likely showed disinhibition (28.6\%) and aberrant motor behavior (14.3\%). The PPA symptoms present in the initial 5 years, and those that develop afterwards, are consistent with previous reports [7]. These findings suggest that, as PPA progresses, the neurodegenerative process expands and eventually involves the brain regions affecting behavior, such as the frontal lobe $[2,18,25]$. 
Singh et al.: Neuropsychiatric Symptoms in Primary Progressive Aphasia and Apraxia of Speech

Table 6. NPS according to disease duration

\begin{tabular}{|c|c|c|c|c|c|c|}
\hline & \multicolumn{3}{|l|}{ PPA } & \multicolumn{3}{|l|}{ PAOS } \\
\hline & $\mathrm{SD}(\mathrm{n}=51)$ & $\mathrm{LD}(\mathrm{n}=14)$ & $\mathrm{p}$ value & $\mathrm{SD}(\mathrm{n}=23)$ & $\mathrm{LD}(\mathrm{n}=9)$ & $\mathrm{p}$ value \\
\hline Delusion & $1(1.9 \%)$ & $1(7.1 \%)$ & 0.3670 & 0 & 0 & $\mathrm{~N} / \mathrm{A}$ \\
\hline Hallucination & $2(3.9 \%)$ & 0 & 0.3203 & $1(3.1 \%)$ & 0 & 0.4783 \\
\hline Agitation & $9(17.6 \%)$ & $3(21.4 \%)$ & 0.7501 & $7(21.9 \%)$ & $1(11.1 \%)$ & 0.4491 \\
\hline Depression & $24(47.1 \%)$ & $4(28.6 \%)$ & 0.0431 & $10(31.2 \%)$ & $3(33.3 \%)$ & 0.9058 \\
\hline Anxiety & $18(35.3 \%)$ & $6(42.9 \%)$ & 0.6060 & $7(21.9 \%)$ & $2(22.2 \%)$ & 0.9823 \\
\hline Euphoria & $2(3.9 \%)$ & 0 & 0.3203 & 0 & 0 & $\mathrm{~N} / \mathrm{A}$ \\
\hline Apathy & $18(35.3 \%)$ & $2(14.3 \%)$ & 0.1114 & $15(46.9 \%)$ & $5(55.5 \%)$ & 0.0472 \\
\hline Disinhibition & $5(9.8 \%)$ & $4(28.6 \%)$ & 0.0358 & $1(3.1 \%)$ & $1(11.1 \%)$ & 0.3700 \\
\hline Irritability & $22(43.1 \%)$ & $6(42.9 \%)$ & 0.9850 & $15(46.9 \%)$ & $4(44.4 \%)$ & 0.8971 \\
\hline Aberrant motor behavior & $1(1.9 \%)$ & $2(14.3 \%)$ & 0.0439 & $5(15.6 \%)$ & 0 & 0.0212 \\
\hline Nighttime behavior & $3(5.9 \%)$ & $1(7.1 \%)$ & 0.8643 & $1(3.1 \%)$ & $1(11.1 \%)$ & 0.3700 \\
\hline Appetite & $8(15.7 \%)$ & $4(28.6 \%)$ & 0.2903 & $1(3.1 \%)$ & $1(11.1 \%)$ & 0.3700 \\
\hline
\end{tabular}

$\mathrm{N} / \mathrm{A}=$ Not applicable; $\mathrm{SD}=$ short duration $(<5$ years $) ; \mathrm{LD}=$ long duration $(\geq 5$ years $)$.

Another interesting finding that has not previously been reported was the fact that some of the behavioral symptoms present early in the disease ( $<5$ years) appeared to resolve over time. For example, aberrant motor behaviors, while present in $15.6 \%$ of the PAOS subjects $<5$ years after onset, were no longer present after 5 years of illness duration. The details of the NPS present before and after 5 years for both PAOS and PPA are shown in table 6 .

The NPS associated with both PPA and PAOS may have treatment implications for physicians as well as for patients and their caregivers. With better knowledge of the NPS associated with these neurodegenerative disorders, appropriate psychosocial education and support can be outlined for both patients and their caregivers and may help them in handling the behaviors. Patients with PPA might also have emotion recognition disturbances that are mediated by attentional deficits [26]. Hence, with a better knowledge of the relationship between illness duration and the appearance of a specific NPS, physicians are better able to discuss long-term outcomes and treatment options.

This study has some limitations. Although there have been studies showing the reliability and validity of the NPI-Q [11,12], the NPI-Q is a brief measure of symptom assessment asking just one question per symptom domain. The simple and brief nature of the NPI-Q may not necessarily capture the nuances of some particularly complex symptoms, particularly during the earlier stages of the illness. For example, it is difficult to know whether patients present with mild depression/anxiety due to an emotional response secondary to language impairment, or due to a noncognitive manifestation of the neurodegenerative process. Another limitation is that the estimation of disease duration is subjective, and we could not follow the progression of behavioral symptoms. Hence, further studies are needed to access the potential mechanisms linking NPS with these disorders, and long-term follow-up of subtypes of PPA and PAOS patients may help in understanding the natural history and evolution of their NPS.

In conclusion, the result of this study highlights the differences in the NPS between the two categories of neurodegenerative speech disorders, PAOS and PPA as well as among the different subtypes of PPA patients. Mood symptoms like anxiety and appetite changes are more likely to be present at initial stages of PPA, whereas behavioral symptoms like aberrant motor behavior and apathy are likely to occur in PAOS. Additionally, it appears that NPS 
Singh et al.: Neuropsychiatric Symptoms in Primary Progressive Aphasia and Apraxia of Speech

evolve with the progression of the disease and may not always be present. A better understanding of the NPS among these groups of patients helps to better outline a treatment strategy for both the patient and the caregiver.

\section{Acknowledgment}

\section{This study was supported by NIH R01-DC010367.}

\section{Disclosure Statement}

T.D. Singh reports no disclosures. J.R. Duffy is a coinvestigator for R01-DC012519 and R01-DC010367. E.A. Strand is a coinvestigator for R01-DC012519 and R01-DC010367. M.M. Machulda is funded by R01-AG037491 (Co-I), R01-DC012519 (Co-I) and the Alzheimer's Association (Co-I). J.L. Whitwell is funded by R01-DC012519 (PI), R01-DC010367 (Co-I), R01-AG037491 (Co-I) and the Alzheimer's Association (PI). K.A. Josephs is funded by NIH R01-DC010367 (PI), R01-AG037491 (PI), R01-DC012519 (Co-I) and the Alzheimer's Association (Co-I).

\section{References}

1 Mesulam MM: Slowly progressive aphasia without generalized dementia. Ann Neurol 1982;11:592-598.

2 Bonner MF, Ash S, Grossman M: The new classification of primary progressive aphasia into semantic, logopenic, or nonfluent/agrammatic variants. Curr Neurol Neurosci Rep 2010;10:484-490.

3 Mesulam MM: Primary progressive aphasia: a 25-year retrospective. Alzheimer Dis Assoc Disord 2007;21:S8S11.

4 Gorno-Tempini ML, Hillis AE, Weintraub S, Kertesz A, Mendez M, Cappa SF, Ogar JM, Rohrer JD, Black S, Boeve BF, Manes F, Dronkers NF, Vandenberghe R, Rascovsky K, Patterson K, Miller BL, Knopman DS, Hodges JR, Mesulam MM, Grossman M: Classification of primary progressive aphasia and its variants. Neurology 2011; 76:1006-1014.

5 Josephs KA, Duffy JR, Strand EA, Machulda MM, Senjem ML, Lowe VJ, Jack CR Jr, Whitwell JL: Syndromes dominated by apraxia of speech show distinct characteristics from agrammatic PPA. Neurology 2013;81:337-345.

-6 Josephs KA, Duffy JR, Strand EA, Machulda MM, Senjem ML, Master AV, Lowe VJ, Jack CR Jr, Whitwell JL: Characterizing a neurodegenerative syndrome: primary progressive apraxia of speech. Brain 2012;135:15221536.

7 Banks SJ, Weintraub S: Neuropsychiatric symptoms in behavioral variant frontotemporal dementia and primary progressive aphasia. J Geriatr Psychiatry Neurol 2008;21:133-141.

-8 Fatemi Y, Boeve BF, Duffy J, Petersen RC, Knopman DS, Cejka V, Smith GE, Geda YE: Neuropsychiatric aspects of primary progressive aphasia. J Neuropsychiatry Clin Neurosci 2011;23:168-172.

-9 Marczinski CA, Davidson W, Kertesz A: A longitudinal study of behavior in frontotemporal dementia and primary progressive aphasia. Cogn Behav Neurol 2004;17:185-190.

10 Rohrer JD, Warren JD: Phenomenology and anatomy of abnormal behaviours in primary progressive aphasia. J Neurol Sci 2010;293:35-38.

11 Cummings JL, Mega M, Gray K, Rosenberg-Thompson S, Carusi DA, Gornbein J: The neuropsychiatric inventory: comprehensive assessment of psychopathology in dementia. Neurology 1994;44:2308-2314.

12 Kaufer DI, Cummings JL, Ketchel P, Smith V, MacMillan A, Shelley T, Lopez OL, DeKosky ST: Validation of the NPI-Q, a brief clinical form of the neuropsychiatric inventory. J Neuropsychiatry Clin Neurosci 2000;12:233239.

13 Duffy J: Apraxia of speech in degenerative neurologic disease. Aphasiology 2006;20:511-527.

14 Josephs KA, Duffy JR, Strand EA, Whitwell JL, Layton KF, Parisi JE, Hauser MF, Witte RJ, Boeve BF, Knopman DS, Dickson DW, Jack CR Jr, Petersen RC: Clinicopathological and imaging correlates of progressive aphasia and apraxia of speech. Brain 2006;129:1385-1398.

15 Josephs KA, Boeve BF, Duffy JR, Smith GE, Knopman DS, Parisi JE, Petersen RC, Dickson DW: Atypical progressive supranuclear palsy underlying progressive apraxia of speech and nonfluent aphasia. Neurocase 2005;11: 283-296.

16 Burrell JR, Hodges JR, Rowe JB: Cognition in corticobasal syndrome and progressive supranuclear palsy: a review. Mov Disord 2014;29:684-693.

17 Josephs KA, Whitwell JL, Eggers SD, Senjem ML, Jack CR Jr: Gray matter correlates of behavioral severity in progressive supranuclear palsy. Mov Disord 2011;26:493-498. 
18 Josephs KA, Duffy JR, Strand EA, Machulda MM, Senjem ML, Lowe VJ, Jack CR Jr, Whitwell JL: Syndromes dominated by apraxia of speech show distinct characteristics from agrammatic PPA. Neurology 2013;81:337-345.

19 Josephs KA, Xia R, Mandrekar J, Gunter JL, Senjem ML, Jack CR Jr, Whitwell JL: Modeling trajectories of regional volume loss in progressive supranuclear palsy. Mov Disord 2013;28:1117-1124.

20 Josephs KA, Duffy JR, Strand EA, Machulda MM, Senjem ML, Gunter JL, Schwarz CG, Reid RI, Spychalla AJ, Lowe VJ, Jack CR Jr, Whitwell JL: The evolution of primary progressive apraxia of speech. Brain 2014;137:27832795.

21 Snowden JS, Bathgate D, Varma A, Blackshaw A, Gibbons ZC, Neary D: Distinct behavioural profiles in frontotemporal dementia and semantic dementia. J Neurol Neurosurg Psychiatry 2001;70:323-332.

22 Starkstein SE, Robinson RG: Mood disorders in neurodegenerative diseases. Semin Clin Neuropsychiatry 1996;1:272-281.

23 Medina J, Weintraub S: Depression in primary progressive aphasia. J Geriatr Psychiatry Neurol 2007;20:153160.

24 Gorno-Tempini ML, Dronkers NF, Rankin KP, Ogar JM, Phengrasamy L, Rosen HJ, Johnson JK, Weiner MW, Miller BL: Cognition and anatomy in three variants of primary progressive aphasia. Ann Neurol 2004;55: 335-346.

25 Harris JM, Gall C, Thompson JC, Richardson AM, Neary D, du Plessis D, Pal P, Mann DM, Snowden JS, Jones M: Classification and pathology of primary progressive aphasia. Neurology 2013;81:1832-1839.

-26 Chow TW, Fridhandler JD, Binns MA, Lee A, Merrilees J, Rosen HJ, Ketelle R, Miller BL: Trajectories of behavioral disturbance in dementia. J Alzheimers Dis 2012;31:143-149.

27 Kumfor F, Miller L, Lah S, Hsieh S, Savage S, Hodges JR, Piguet O: Are you really angry? The effect of intensity on facial emotion recognition in frontotemporal dementia. Soc Neurosci 2011;6:502-514. 